\title{
Characterization of Protoporphyrin IX Species In Vitro Using Fluorescence Spectroscopy and Polar Plot Analysis
}

Kai Wen Teng ${ }^{1,2, *}$, Sang Hak Lee ${ }^{2,3, *}$

1. Center for Biophysics and Computational Biology, 2. Department of Physics, University of Illinois of Urbana-Champaign, Urbana, IL 61801, USA 3. Department of Chemistry, Pusan National University, Busan 46241, Korea

* Corresponding author: teng5@illinois.edu and shlee77@pusan.ac.kr

\section{Supporting Information}

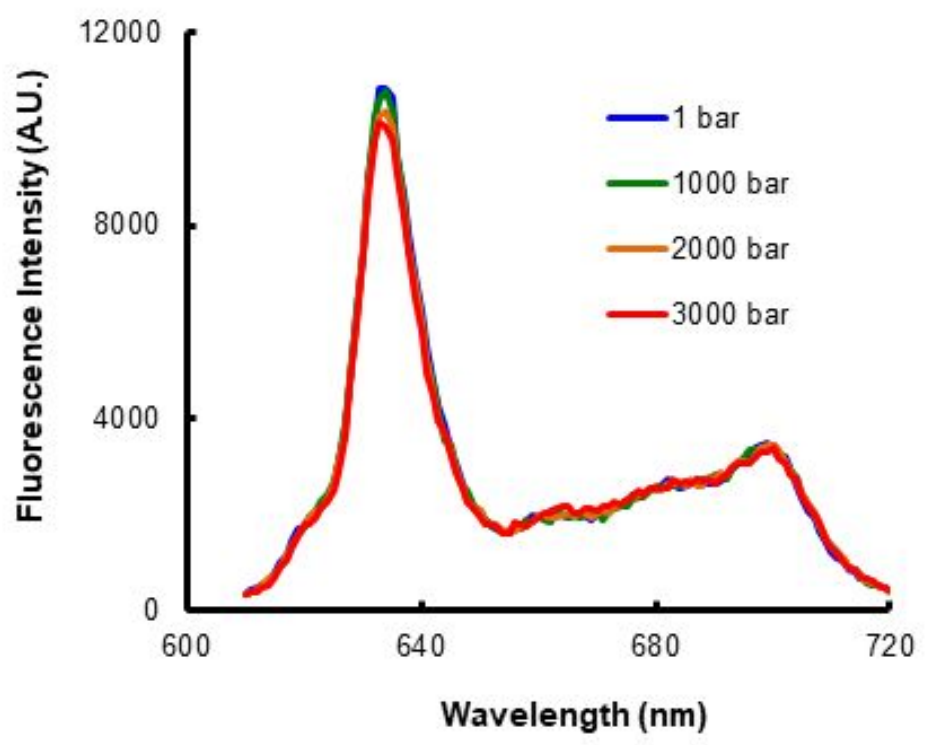

Figure S1. Emission spectra of PPIX in 0.6\% Triton X-100 under high pressure. As pressure varied from 1 to 3000 bar in 1000 bar increments, little to no change was observed in the emission spectrum. 


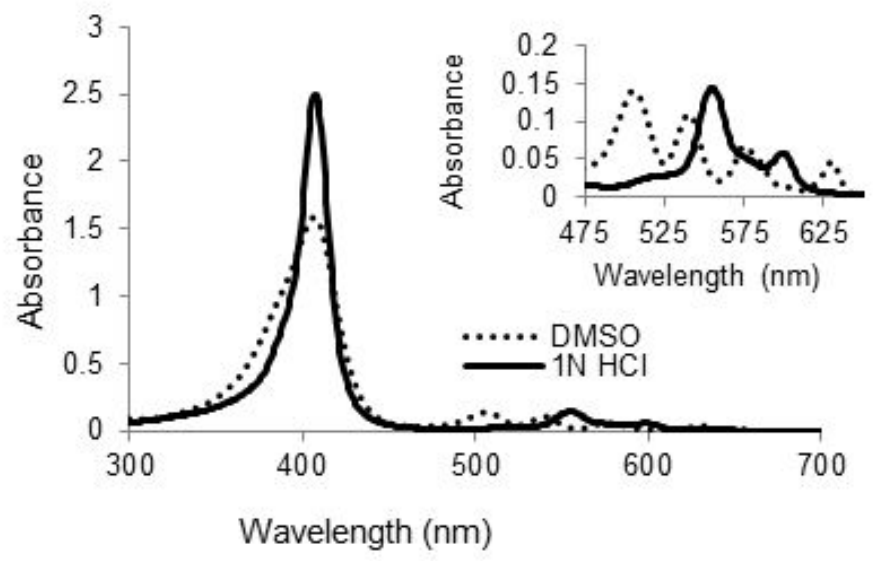

Figure S2. Absorbance Spectra of PPIX Dication Compared to Neutral PPIX. The q-bands of the absorbance spectra (inset) are shown for PPIX in $1 \mathrm{~N} \mathrm{HCl}$ and PPIX in DMSO. PPIX in $1 \mathrm{~N}$ $\mathrm{HCl}$ shows 2 q-bands as oppose to 4 q-bands in the DMSO. 